\title{
Effects of Nitrogen incorporation and thermal annealing on the optical and spin properties of GaPN dilute nitride alloys
}

M. A. G. Balanta ${ }^{1,4}$ P. B. A. de Oliveira ${ }^{1}$, H Albalawi $^{2,8}$, Y. Galvão Gobato ${ }^{1, *}$, H. V. A. Galeti ${ }^{3}$, A.D.Rodrigues ${ }^{1}$, M.Henini ${ }^{2}$, S. Almosni ${ }^{5}$, C. Robert ${ }^{6}$, A. Balocchi ${ }^{6}$, Y. Léger ${ }^{5}$, H. Carrère ${ }^{6}$, M. Bahri ${ }^{7}$, G. Patriarche $^{7}$, X. Marie ${ }^{6}$, C. Cornet ${ }^{5}$

\author{
${ }^{I}$ Physics Department, Federal University of São Carlos,13560-905, São Carlos, SP, Brazil \\ ${ }^{2}$ School of Physics and Astronomy, University of Nottingham, Nottingham NG7 2RD, UK \\ ${ }^{3}$ Electrical Engineering Department, Federal University of São Carlos, 13560-905, São Carlos, SP, Brazil \\ ${ }^{4}$ Universidade Federal de Uberlândia-ICENP, 38304-402, Ituiutaba, MG, Brazil \\ ${ }^{5}$ Univ Rennes, INSA Rennes, CNRS, Institut FOTON - UMR 6082, F-35000 \\ Rennes, France
}

${ }^{6}$ Université de Toulouse, INSA-CNRS-UPS, LPCNO, 135 avenue de Rangueil, 31077 Toulouse, France

${ }^{7}$ Centre de nanosciences et de Nanotechnologies (C2N), CNRS, Université Paris Sud, Université Paris Saclay, 10 Boulevard Thomas Gobert, 91120 Palaiseau, France.

${ }^{8}$ Physics Department, Faculty of science, Princess Nourah Bint Abdulrahman University, Riyadh, Saudi Arabia.

* Also at High Field Magnet Laboratory (HFML - EMFL), Radboud University, Toernooiveld 7, 6525 ED NIJMEGEN, The Netherlands.

Here, we report on the structural, optical and magneto-optical properties of as-grown and thermal annealed GaPN thin films containing different $\mathrm{N}$ concentrations grown by Solid-Source Molecular Beam Epitaxy on GaP substrates. A wide range of characterization techniques were used to investigate the effect of $\mathrm{N}$ incorporation and thermal annealing effect of GaPN/GaP epilayers for a $N$ content between $0.5 \%$ and $3 \%$. Our results have shown that as we increase the $\mathrm{N}$ content the PL peak energy presents a red shift which is explained by a band gap reduction. However, important effects of exciton localization on optical properties were also evidenced from $\mathrm{cw}$ and time resolved PL (PLRT) and magneto-PL. Our results have demonstrated that as the concentration of $\mathrm{N}$ is increased, exciton localization effect is enhanced. A substantial improvement of the optical quality and spin properties of the epilayers has been observed after thermal annealing which are significantly more important for samples with higher nitrogen contents. 


\section{Introduction}

III-V dilute nitride alloys have recently attracted a great of attention for applications in optoelectronics particularly for solar cells [1-5]. Among these alloys, GaPN is an interesting material for pseudomorphic integration of photonics with silicon [6,7]. Furthermore, recently it was found that GaNPbased nanowires are a promising material for innovative device applications due to their beneficial properties related to their architecture [8-11]. Gallium phosphide $(\mathrm{GaP})$ has the smallest lattice mismatch with silicon $(0.37 \%$ at room temperature) amongst all binary III-V compound semiconductors. In addition, the residual strain due to this lattice mismatch can be compensated by the incorporation of a few per cent of nitrogen $(\mathrm{N})$ in $\mathrm{GaP}[12,13]$. It is therefore important to investigate the effect of incorporation of $\mathrm{N}$ in GaPN in order to obtain high crystal quality and lattice matched heteroepitaxial GaPN thin layers on silicon substrates for applications in optoelectronics and photovoltaics. In fact, the doping of GaP semiconductor, which is an indirect bandgap semiconductor, with $(\mathrm{N})$ isoelectronic impurity has been widely used in the past to improve its radiative efficiency, and thus, making it suitable for optoelectronic applications. It has been shown that the incorporation of $N$ in GaP leads to an extremely large band-gap bowing and $\mathrm{N}$-induced transformation from an indirect to a pseudo-direct band gap which consequently increases the emission intensity of the semiconductor. It was also previously shown that the incorporation of small content of N into P-rich GaPAs leads to the formation of intermediate band and the change of the nature of the fundamental band gap from indirect to direct [14]. The observed $\mathrm{N}$-induced modification of the electronic band structure was also explained by the Band Anti Crossing (BAC) model. The changes of band structure is usually described by this model that calculates the changes of band structure induced by isovalent $\mathrm{N}$ atom in a III-V host matrix. According to this model, the interaction of the localized $\mathrm{N}$ level with the states of the GaPAs or GaP host leads to the formation of E- and E+ bands in GaNP or GaPN alloy, respectively [14]. In addition, the observation of the isolated intermediate band offers a potential of using P-rich GaNPAs or GaP alloys for intermediate band solar cells [15,16]. However, GaPN semiconductors have many non-radiative recombination channels, mainly 
associated with the formation of defects, such as vacancies, interstitials, and impurity atoms that have a tendency to increase with the increasing of the $\mathrm{N}$ content. [17-20]. Particularly, the optical quality can be improved if the density of defects are reduced which can be realized by optimizing the growth conditions or by performing post-growth annealing $[21,22]$. In the present work, we explored this effect by investigating as-grown and thermal annealed GaPN samples containing different $N$ contents between 0.5 and $3 \%$. The effect of thermal annealing was not previously investigated for GaPN layers. In addition, we present for the first time magneto-optical results for GaPN layers under high magnetic fields for as-grown and annealed samples. Structural properties were studied by high resolution X-ray diffraction and Raman scattering. Optical properties were analysed by photoluminescence ( $P L)$, photoluminescence excitation (PLE) and time resolved photoluminescence (TRPL). In general, our results have also revealed the observation of the isolated intermediate band at low $\mathrm{N}$ concentration and important effects of exciton localization and improvement of the optical properties after thermal annealing of the GaPN layers which are essential for potential manufacture of GaPN based semiconductors devices. In addition, a gexc factor of $\sim 2$ was observed which is similar for all the samples studied.

\section{Experimental Details}

In this work, the samples were grown by Solid-Source Molecular Beam Epitaxy (SSMBE) using the apparatus described in ref. [23]. Phosphorus and atomic $\mathrm{N}$ were generated with a valved cracker cell and a valved RF plasma $\mathrm{N}_{2}$ cell operating at a $400 \mathrm{~W}$ power and with a $0.5 \mathrm{sccm}$ high purity $\mathrm{N}_{2}$ flow rate, respectively. Most of the samples have nominally the same structure, i.e. after the oxide desorption performed at $650{ }^{\circ} \mathrm{C}$, a $100 \mathrm{~nm}$-thick GaP buffer layer is grown at $580^{\circ} \mathrm{C}$, followed by a $100 \mathrm{~nm}$-thick GaPN epilayer grown at $480^{\circ} \mathrm{C}$. Finally, a $10 \mathrm{~nm}$ GaP cap layer was deposited, in order to avoid surface contribution to the optical properties of GaPN. Specific samples (with similar growth conditions) were grown for detailed annealing studies by TRPL and transmission electron microscopy, where the GaPN active layers are $50 \mathrm{~nm}$ - 
thick and the GaP cap layers are $100 \mathrm{~nm}$, in order to study structurally the $\mathrm{N}$ exodiffusion during annealing. The $\mathrm{N}$ composition in the GaPN layer was controlled mainly by the growth rate, $\mathrm{V} / \mathrm{III}$ beam equivalent pressure ratio and $\mathrm{N}$ plasma cell valve opening, as described in details in ref. [23]. The GaP substrates were carefully selected to avoid $\mathrm{N}$ incorporation inhomogeneities due to roughening [24]. The $N$ content was deduced from standard $\theta-2 \theta$ high resolution X-ray diffraction (HRXRD) for the different samples, assuming a linear dependency of the GaPN lattice parameter with the $\mathrm{N}$ composition, as already shown in ref. 7 and 22 for similar thicknesses. In the following, the $\mathrm{N}$ contents measured for each sample are given with the corresponding experimental data. The GaPN layer thickness is limited to $100 \mathrm{~nm}$ in order to avoid plastic relaxation. Rapid Thermal Annealing (RTA) was performed at $800^{\circ} \mathrm{C}$ for 5 minutes in a $\mathrm{N}_{2}$ and $\mathrm{H}_{2}$ mixture atmosphere for some of the samples studied. PL measurements were performed at $10 \mathrm{~K}$ using a Janis closed-cycle refrigerator cryostat and Andor $0.5 \mathrm{~m}$ spectrometer coupled with $\mathrm{Si}$ CCD. Polarization resolved PL measurements under magnetic field were performed at $2 \mathrm{~K}$ in a $15 \mathrm{~T}$ Oxford Magnet with the magnetic field parallel to the growth direction (Faraday configuration). A linearly-polarized blue line (488 $\mathrm{nm}$ ) from a continuous-wave $\mathrm{Ar}+$ laser was used as optical excitation. Therefore, the photogenerated carriers in the GaPN layers should not present any preferential spin polarization. Right $\left(\sigma^{+}\right)$and left $\left(\sigma^{-}\right)$circularly-polarized PL signals were selected with appropriate optical components (achromatic quarter wave plate and Glan-Thompson Calcite Polarizer). Raman scattering measurements at room temperature were performed using 488nm laser line and recorded with a triple grating spectrometer coupled with CCD detector. Experimental parameters were set to furnish a spectral resolution $\sim 1.5 \mathrm{~cm}^{-1}$. PLE measurements were carried out in a cold finger cryostat at low temperature $(\sim 10 \mathrm{~K})$. A $50 \mathrm{~W}$ tungsten halogen lamp combined with a 1-m single grating monochromator served as tunable light source for both PL and PLE measurements. The emission from the layers was dispersed by a $0.75-\mathrm{m}$ double monochromator and detected with a liquid- $\mathrm{N}$ cooled S1-photomultiplier. TRPL measurements were performed using a pulse-picker to decrease the Titanium-Sapphire laser repetition frequency to $4 \mathrm{MHz}$ which is placed before a non-linear crystal to double the fundamental Ti:Sa emission energy to $3.1 \mathrm{eV}$ 
$(400 \mathrm{~nm})$ in order to excite $\mathrm{GaP}\left(\mathrm{E}_{\mathrm{GAP}}=2.27 \mathrm{eV}\right)$. The laser light was then focussed to a $100 \mu \mathrm{m}$ spot diameter with a time-averaged power around 0.5 $\mathrm{mW}$. The PL signal is detected by using a Hamamatsu S1 streak camera. The $\mathrm{PL}$ decay times are then fitted using a double exponential decay function. STEM-HAADF (High-angle annular dark-field scanning transmission electron microscopy) measurements were performed using a Cs corrected TEM/STEM Jeol2200Fs. The microscope was operated at $200 \mathrm{KV}$. The beam convergence angle was $30 \mathrm{mrad}$. For the HAADF detector, the collection angles used were $62.3 \mathrm{mrad}$ and $170 \mathrm{mrad}$ for the internal and external angles, respectively.

\section{Results and Discussion}

\subsection{Photoluminescence general properties}

Figure 1 (a) shows the typical PL spectra obtained for different $\mathrm{N}$ concentrations at $300 \mathrm{~K}$. In general, it was observed a red-shift of the PL energy peak with the increase of $\mathrm{N}$ content, as shown in Fig. 1 (b). This expected behavior is usually associated with the giant band gap bowing effect in dilute nitride alloys [25-32]. It is well known that $\mathrm{N}$ forms perturbed host conduction states which shift to lower energy when increasing the $N$ content. In Fig. 1(b), bandgap energies calculated with tight-binding (TB) simulations are superimposed with experimental data, showing an excellent agreement. A $50 \mathrm{meV}$ systematic shift was added to TB bandgaps that are performed nominally at $0 \mathrm{~K}$. However, the incorporation of $\mathrm{N}$ in GaP also introduces important disorder effects which affect considerably the optical properties. These effects will be investigated later in this paper by different techniques such as Raman Spectroscopy, power and temperature dependent PL, TRPL and PLE. Figure 2 shows typical PL spectra for different laser power at low temperature (10K). For all samples, different broad bands were observed at lower energies and sharp PL peaks at higher energies $(>2 \mathrm{eV})$ depending on laser power. These broad bands at lower energy are usually associated with exciton localization effects. The observed sharp emissions are due to NNi (nitrogen-nitrogen interstitial) centers. Actually, these sharp peaks are usually observed in the doping regime at low 
incorporation of $\mathrm{N}$ in GaP and in non-intentionally doped $\mathrm{GaP}$ due to residual doping. In GaP crystal, all these states are localized below the conduction band. In our case, the observed sharp lines are associated with the emission in the GaP substrate. In addition, the observed asymmetry of the broad band is usually attributed with disorder effects such as random fluctuations in alloy composition which could result in a smearing of band edges and in an extended density of states into the band gap. This asymmetry of the PL spectrum is well known for dilute nitrides. Due to the fact that the high energy tail is enhanced by thermal filling of the bands, the asymmetric shape is more pronounced at lower temperatures than at room temperature and higher laser power. Particularly, it was observed a blue shift of the PL band with increasing laser power and a reduction of band tail at lower energy. Furthermore, it was also found (not shown here) that the integrated PL intensity has a nearly linear variation as a function of excitation density. These features are usually explained by a gradual filling of energy states resulting from localized centers particularly due to alloy fluctuations in the low energy band tail. The radiative recombinations associated with the band tail are expected to be the dominant mechanism which would be responsible for this laser power dependence of PL spectrum. We have investigated the temperature dependence of GaPN layers with different $N \%$ content as shown in figure 3 . Figure 3 (a) shows the temperature dependence of PL peak energy. An anomalous behavior of PL peak position versus $\mathrm{T}$ was observed. Particularly, the PL peak energy cannot be fitted by the Varshni equation. This behavior, which is similar to the reported results on other dilute nitride such as GaAsN and InGaAsN, evidences important effects of localization of excitons by disorder for all investigated GaPN layers. In general, it is well known that for dilute nitrides the temperature dependence of PL peak show an $\mathrm{S}$-shape behavior [33-38]. However, only for the $2.7 \% \mathrm{~N}$ content it was possible to clearly evidenced this behavior, this result suggests a high carrier localization on this sample, we reinforces this fact with the next results. In order to improve the optical quality of our GaPN samples, we have performed thermal annealing treatment. Figure $3 \mathrm{~b}$ shows a typical temperature dependence of $\mathrm{PL}$ peak energy for annealed samples using laser excitation at $488 \mathrm{~nm}$ and $30 \mathrm{~mW}$. A typical S-shape dependence of PL peak versus temperature was observed after annealing. Figure 4 illustrates a typical PL spectrum for $1.4 \% \mathrm{~N}$ at $300 \mathrm{~K}$ 
before and after thermal annealing at $800^{\circ} \mathrm{C}$ and $5 \mathrm{mins}$. An important improvement of $\mathrm{PL}$ intensity for all samples was ascertained. Particularly for the $1.4 \% \mathrm{~N}$ content this enhancement of PL intensity is about 3 times after thermal annealing. In addition, an important blue shift of PL spectrum after thermal annealing was determined. This result is in agreement with previous reports $[39,40]$. Usually, at lower temperatures the PL spectrum is dominated by recombination of localized excitons. Above $200 \mathrm{~K}$, the PL peak usually presents a blue shift and an increase of PL linewidth. At this condition, the localized carriers have enough thermal energy and starts to escape to the quasidelocalized states. As mentioned before this effect was typically observed in dilute nitrides and is usually called the S-shape behavior.

\subsection{Structural properties}

Before discussing in details the impact of annealing and $\mathrm{N}$ composition on advanced optical properties, structural investigations have been performed by Raman spectroscopy and transmission electron microscopy to find out the main physical processes involved during annealing. Fig. 5(a), presents the Raman spectra at $300 \mathrm{~K}$ for GaPN layers with different $\mathrm{N}$ concentrations. The most intense peak at around $402 \mathrm{~cm}^{-1}$ is related to GaP LO-phonons. At $365 \mathrm{~cm}^{-1}$ is the TO-phonons peak, which is forbidden by Raman selection rules in backscattering geometry, but in this case they are activated by disorder. Spectral features with wave-numbers lower than $300 \mathrm{~cm}^{-1}$ are associated with the density of states (DOS) of acoustic phonons. It is noticed that the LOphonon peaks (inset of Fig. 5 (a)) present the same width for $\mathrm{N}$ contents up to $2.1 \%$, however, a large broadening is observed for $\mathrm{N}=2.7 \%$. Generally, peak broadening occurs as a consequence of a breakdown in the selection rule for the momentum conservation. In the presence of crystalline disorder, phonons become localized. The decrease in the uncertainty of the phonon localizations corresponds to an increasing in the uncertainty of their momenta, allowing phonons far from the Brillouin Zone center to contribute to the scattering process. Since GaP LO phonons present negative dispersion relation [41], the resulting spectral line will be broadened towards lower wavenumbers, indicating 
that the $\mathrm{N}$ incorporation has induced strong structural disorder in the layer with higher $\mathrm{N}$ proportion. Raman spectra of the samples after annealing were also measured. In fig 5 (b), the Raman data for the sample with $\mathrm{N}=2.1 \%$, are displayed as an example. No measurable change were found in the spectra taken before and after the annealing process, indicating that the thermal treatment did not produce significant structural modifications. STEM and XRD measurements were performed on similar samples ( $N=2 \%$ but with larger $\mathrm{GaP}$ capping layer and thinner GaPN layer) in order to investigate possible structural changes of these samples after annealing. Two cross-sectional STEM-HAADF images are shown in Fig. 6 before (a) and after (b) the annealing process. Surprisingly, GaPN layer has higher HAADF intensity compared to GaP ones. It worth noting that the HAADF image mode is also called Z-contrast imaging. It is proportional to the square of the atomic number. Thus, the heavier the elements the higher the contrast, that should lead to a larger HAADF intensity for the $\mathrm{GaP}$. This effect has been reported recently in $\mathrm{GaAs} / \mathrm{GaAsN}$ layers $[42,43]$. It is here attributed to the static atomic displacement effect [44]. The corresponding contrast profiles are also given along the growth direction (Fig.6(c)). From this figure, we can conclude that as-grown and annealed GaPN/GaP interfaces are sharp and well-defined and that structural inhomogeneity is not observed inside the GaPN epilayers, as confirmed also by X-ray diffraction experiments, see ref. 22. From the important findings that no modification of the GaPN is observed during the annealing, keeping the same interface quality and HAADF contrast, we evidence that exodiffusion of $\mathrm{N}$ out of the GaPN layers did not occur during the annealing process. This tends to indicate that structural non-radiative defects that are impacted by annealing are not related to $\mathrm{N}$ local inhomogeneities, but more probably to Ga-vacancies induced during the growth-process, which also explains why the impact of annealing cannot be clearly seen from X-ray diffraction and Raman experiments.

\subsection{PLE results}

Figure 7(a) shows the PL (open circles and solid square) and PLE (solid line) spectra at $10 \mathrm{~K}$ for the as-grown and annealed samples for $\mathrm{N}=1.4 \%$. We have observed that the PLE spectra is dominated by a broad band centered at $\sim 2.3$ 
$\mathrm{eV}$. This broad band has been reported previously $[45,46]$ for $\mathrm{N}$ concentration up to $0.56 \%$ and was associated with a miniband created by the coupling between $\mathrm{N}$-levels and the conduction band. For low N\% concentration a PLE peak associated with an exciton bound to an isolated $\mathrm{N}$ atom is usually observed and labelled $\mathrm{A}$-line. By increasing the $\mathrm{N}$ concentration this line results in a broader band. A shoulder at $\sim 2.2 \mathrm{eV}$ was also observed and attributed to the isoelectronic traps formed by NN pairs (NN1). The PLE spectra were also measured for several detection energies (not shown here) for both samples and showed a similar result indicating that the emission and absorption bands are due to the same layer. In addition, a large energy separation between the PL and PLE peaks was observed. Particularly, a Stokes Shift of $\sim 190 \mathrm{meV}$ was seen for $N=1.4 \%$ as-grown sample. Since the PLE spectra are related to the density of states, the high density of the continuous states may dominate, whereas the radiative transitions occur for the occupied states, at lower energy (localized states). This effect explains the large energy separation between absorption and emission observed in Fig. 7(a), which is typical for GaPN layers. After thermal annealing, this energy separation was drastically reduced to $\sim 130$ $\mathrm{meV}$, suggesting that thermal annealing reduces the localized states, possibly due to defects and potential fluctuations, resulting in a PL blue shift and a decreasing of the PL broadening. However, it was observed that the peak position of the PLE band was not affected by the thermal annealing. On the other hand, the PLE band width was reduced which could also be related to the reduction of the localized states in the sample. Considering that the $\mathrm{N}$ states become a mini-band in the gap, it is reasonable to consider a parabolic band around the edge, i.e. the density of states can be described as $A_{0} * \sqrt{E-E_{0}}$ for the minimum energy edge, where $A_{0}$ is a constant and $E_{0}$ is the minimum band edge. Similarly, for maximum edge the density of states can be given by $A_{F} * \sqrt{E_{t}-E}$. where $\mathrm{A}_{F}$ is a constant and $\mathrm{E}_{\mathrm{t}}$ is a threshold band edge. We can extract the absorption edge ( $E_{0}$ and $E_{t}$ ) for each PLE spectra. Considering that the PLE band shown in Fig. 7(a) is related to the lower branch of the impurity mini-band, we can estimate band width by $\left(E_{t}-E_{0}\right)$. The values of $E_{0}, E_{t}$, and $\left(E_{t}\right.$ - $E_{0}$ ) are plotted in figure $7(\mathrm{c})$ as function of $N$ concentration for annealed samples. The value of $E_{t}$ is independent of the $N$ composition, while, as 
expected, $E_{0}$ decreases and $\left(E_{t}-E_{0}\right)$ increases due to the increase of the coupling as $\mathrm{N}$ increases. We also plot the energy separation between $\mathrm{E}_{0}$ and $\mathrm{PL}$ peak (Stokes shift) in Fig. 7(b). The Stokes shift increases from $\mathrm{N}$ content from $1.4 \%$ to $2.1 \%$ and then remains constant. The localization effects seem to have a saturation for large values of $\mathrm{N} \%$.

\subsection{Time Resolved Photoluminescence}

Typical TRPL streak images for a $\mathrm{N}=1 \%$ sample before and after annealing are shown in Figure 8 (a) and (b), respectively, at $\mathrm{T}=10 \mathrm{~K}$. In general, it was observed that the decay time of $\mathrm{GaP}(\mathrm{N})$ is usually of several nanoseconds probably due to the indirect nature of the recombination. The temporal and spectral evolutions presented in the figures do not change drastically from one sample to the other, the overall shape is always similar. But looking more carefully at the two presented streak images, one can see that annealing slightly impacts the time-resolved PL properties in terms of a narrowing of the spectrum and modification of the radiative lifetime. From streak images, PL decay times are fitted by using a double exponential decay function, $\mathrm{T}_{1}$ and $\mathrm{T}_{2}$ being the associated lifetimes. The origin of these two decay times could be ascribed to: (1) delocalised state emissions associated with shorter decay times and (2) emissions associated with localised density of states (longer decay times). Both of them being impacted by the presence of non-radiative centers. Figure 8 (c) and (d) present the PL decay times measured for $\mathrm{N}=1 \%$ sample before and after annealing. In this case, beyond the spectral narrowing clearly observed in the annealed sample, the decay time evolution does not change significantly, and a small decrease of the $\mathrm{T}_{2}$ value at $10 \mathrm{~K}$ is even measured, as compared to the as-grown samples. It is then concluded that in this range of compositions, the annealing mainly plays an important role on the homogeneity of the samples, but does not impact significantly on non-radiative recombination contributions. It was also observed that the longer decay times are more sensitive to thermal annealing. Particularly, a characteristic S-shape as a function of the temperature was observed after annealing, similar to the behaviour usually observed for the temperature variation of the PL peak in 
dilute nitrides. This result points to the existence, after annealing, of shallower and fewer $\mathrm{N}$-induced defects which can be ionised at relatively low temperature: an increase of the decay time is observed starting from a temperature as low as $40 \mathrm{~K}$, in parallel to a symmetric increase of the band gap (not shown). The same analysis is now performed on samples having $\mathrm{N}=2 \%$. Decay times for asgrown and annealed samples are reported in fig. 9 (a) and (b), respectively. In this case, both decay times are always significantly larger after annealing, which evidences an overall improved optical quality of the material. Whereas for the as-grown sample the decay times monotonously decrease with the temperature, for the annealed sample it is clear that temperature-activated defects dominate only above $150 \mathrm{~K}$. These improved optical qualities are confirmed by the observation of a sizeable larger activation energy of the decrease of the PL integrated intensity as a function of temperature (Fig. 9(c)) and a marked overall narrower linewidth (Fig. 9(d)). Therefore, the benefits of post-thermal annealing are significantly more important in samples with high $\mathrm{N}$ contents. All these data are consistent with an improved optical quality of the annealed GaPN layer, a key result for the realisation of buffer layers for the monolithic integration of optoelectronic devices on silicon [47- 50].

\subsection{Magneto-PL}

We have performed a detailed study of magneto-optical properties of GaPN layers. It is important to mention that no results of magnetoluminescence resolved in polarization measurements in GaPN systems have been reported previously. Figure 10 shows typical PL spectra as function of magnetic field at $2 \mathrm{~K}$ and $30 \mathrm{~mW}$ of laser excitation for the sample with $\mathrm{N}=2.1 \%$. It was observed that the diamagnetic shift, which is obtained from the difference between the energy peak under magnetic field and zero magnetic field, is almost zero which evidences important effects of exciton localization in GaPN layers. Similar results were observed in all as-grown and post-thermal annealed GaPN samples with different $N$ concentrations. These results evidence important effect of exciton localization [51,52] in GaPN layers probably due to composition variation which cannot be changed after thermal annealing 
treatment. We have also investigated the polarization resolved PL for as-grown and annealed layers. Figure 11 show typical $\sigma+$ and $\sigma$ - polarization resolved PL spectra for $\mathrm{N}=2.1 \%$ samples (as-grown and annealed) at $2 \mathrm{~K}$ and under $15 \mathrm{~T}$. It was observed that only for $\mathrm{N}=2.7 \%$ sample the polarization increases linearly for all the range of the magnetic field applied. Nevertheless, this behavior was also observed in the other samples at low fields, $\sim B=6 \mathrm{~T}$ and tends to saturate at higher magnetic fields, reaching values up to $~ 40 \%$. A similar behavior was observed in InGaAs quantum well structures where the magnetic field dependence of the polarization was attributed to the fact that it is proportional to the spin polarization of holes once both electron spin levels below $E_{F}$ are equally occupied. At low magnetic fields the polarization of holes is determined by $g_{h} \mu_{B} B /\left(k_{B} T\right)$ and then saturates at high $B$ because of the similar occupation of spin levels for holes [53,54]. Here, $g_{h}$ is the hole g-factor and $\mu_{B}$ is the Bohr magneton. The fact that we found a linear behavior only in the sample with higher content of $\mathrm{N}$, indicates a higher efficiency of the annealing eliminated more significantly the scattering centers for the relaxation of the spin of the holes in this sample. A significant value of odd circular polarization degree for GaPN layers was obtained. In addition, an increase of the degree of polarization was observed after thermal annealing which could be explained by important changes of spin relaxation and recombination times after thermal annealing due to the reduction of defects $[55,56]$. This effect is illustrated in figure 12 which shows the magnetic field dependence of degree of polarization for the as-grown and annealed samples. An interesting effect is observed in these GaPN layers. We remark that the difference between the circular polarization degree of the as-grown samples and annealing samples increases as the $\mathrm{N}$ concentration increases. This result is consistent with the fact that the effect of the annealing must be more efficient in reducing the defects which cause spin relaxation in samples with higher $\mathrm{N}$ concentration. In the diluted limit of the impurity concentration, the annealing is less efficient in decreasing those scattering centers. Moreover, the higher polarization degree is obtained for the sample with $\mathrm{N}=1 \%$ for both as-grown and annealed samples. This fact reinforces the previous results related with the increase of the polarization degree Postthermal annealing which indicates that for this $\mathrm{N}$ concentration, less scattering 
centers for spin relaxation are present in the sample. Finally, we have determined the gexc factor for GaPN layers. The inset of the figure 11 (a) presents the normalized PL spectra for $\sigma+$ and $\sigma-$ emission in order to obtain the spin splitting for sample with $\mathrm{N}=2.1 \%$ at $15 \mathrm{~T}$. From this value we have extracted a gexc factor of $\sim 2$ which is similar for all the samples studied. Despite a relatively high circular polarization of the light emitted in relation to other systems with localized effects, the small spin splitting is related with the fact that the relaxation of the spin via scattering from defects and impurities in this system, is accompanied by a decrease of the Zeeman energy, which requires coupling of the spin system with the lattice [57].

\section{Conclusion}

We have studied the effects of $\mathrm{N}$ incorporation and thermal annealing on the optical, structural and spin properties of GaPN layers. We have evidenced important effects of localization of carriers by disorder. In addition, it was also shown that the annealing process successfully improves the optical qualities of the epilayers in terms of PL intensity, PL decay time, activation energy, linewidth and polarization degree. Nevertheless, no modification of the GaPN structural properties was observed after thermal annealing. In addition, We presented for the first time a study of polarization resolved PL in these systems. The magneto-PL results revealed that the diamagnetic shift is almost zero which also evidences important effects of exciton localization in GaPN layers. A relatively high circular polarization of the light emitted was observed for all samples at high magnetic fields which are higher than the values observed in other systems with localized effects. Finally, our results evidenced an improvement of the optical properties after thermal annealing of the samples which is essential for potential manufacture of GaPN based semiconductors devices.

\section{Acknowledgements}


We acknowledge the Brazilian financial agencies CNPq, CAPES, FAPESP. Y.G.G. and H.V.A.G. acknowledge FAPESP grants 18/01808-5, 16/10668-7 and 14/50513-7. M. A. G. B. and H.V.A.G acknowledge Capes grants 88881.068192/2014-01. M. A. G. B. acknowledge the financial support from PNPD - Capes-PPG- UFSCar. Finally the authors are grateful to Grupo de Propriedades Óticas (GPO) at Universidade Estadual de Campinas (UNICAMP) for PLE and magneto-photoluminescence measurements and to Professor F. likawa for fruitful discussions. 


\section{References}

1. S. Almosni a, P. Rale b, C. Cornet a, M. Perrin a, L. Lombez b, A. Létoublon a , K. Tavernier a , C. Levallois a , T. Rohel a, N. Bertru a , J.F. Guillemoles b,c , O. Durand. Correlations between electrical and optical properties in lattice matched GaAsPN/GaP solar cells. Solar Energy Materials and Solar Cells. Elsevier, 147, pp.53-60 (2016).

2 S. Ilahi, Samy Almosni, Fares Chouchane, Mathieu Perrin, K. Zelazna, N. Yacoubi, R. Kudrawiec, Pierre Râle, Laurent Lombez, Jean-François Guillemoles, Olivier Durand, C. Cornet. Optical absorption and thermal conductivity of GaAsPN absorbers grown on GaP in view of their use in multijunction solar cells. Solar Energy Materials and Solar Cells, Elsevier, 141, pp.291-298 (2015).

3 M. Henini, ed., Dilute Nitride Semiconductors (Amsterdam: Elsevier, 2005)

4 D. Derkacs, R. Jones-Albertus, F. Suarez, and O. Fidaner, J. Latticematched multijunction solar cells employing a $1 \mathrm{eV}$ GalnNAsSb bottom cell.Photonics Energy 2, 021805 (2012).

5 Michael Wiemer, Vijit Sabnis, and Homan Yuen. "43.5\% efficient lattice matched solar cells", Proc. SPIE 8108, High and Low Concentrator Systems for Solar Electric Applications VI, 810804 (2011).

6 K. Kharel, A. Freundlich. (2018). Band structure and absorption properties of $(\mathrm{Ga}, \mathrm{In}) /(\mathrm{P}, \mathrm{As}, \mathrm{N})$ symmetric and asymmetric quantum wells and super-lattice structures: Towards lattice-matched III-V/Si tandem. Journal of Applied Physics 124, 095104 (2018)

7 S. Almosni, C. Robert, T. Nguyen Thanh, C. Cornet, A. Létoublon, T. Quinci, C. Levallois, M. Perrin, J. Kuyyalil, L. Pedesseau, A. Balocchi, P. Barate, J. Even, J. M. Jancu, N. Bertru, X. Marie, O. Durand, and A. Le Corre. Evaluation of InGaPN and GaAsPN materials lattice-matched to Si for multi-junction solar cells. J. Appl. Phys. 113, 123509 (2013).

8 Matthias Steidl, Mingjian Wu, Katharina Peh, Peter Kleinschmidt, Erdmann Spiecker, Thomas Hannappel. Impact of $\mathbf{N}$ Incorporation on VLS Growth of GaP(N) Nanowires Utilizing UDMH. Nanoscale Research Letters 13:1, 417 (2018).

9 Matthias Steidl, Klaus Schwarzburg, Beatriz Galiana, Thomas Kups, Oliver Supplie, Peter Kleinschmidt, Gerhard Lilienkamp, T Hannappel. MOVPE growth of GaP/GaPN core-shell nanowires: $\mathbf{N}$ incorporation, morphology and crystal structure. Nanotechnology 30:10, 104002 (2019).

10 Chen SL, Chen WM, Ishikawa F, Buyanova IA. Suppression of nonradiative surface recombination by $N$ incorporation in GaAs/GaNAs core/shell nanowires. Sci Rep 5:11653 (2015). 
11 Filippov S, Jansson M, Stehr JE, Palisaitis J, Persson POA, Ishikawa F, Chen WM, Buyanova IA. Strongly polarized quantum-dot-like light emitters embedded in GaAs/GaNAs core/shell nanowires. Nanoscale 8:15939-1594 (2016).

12 A. A. Lazarenko, E. B. Nikitina, M. C. Sobolev, E. B. Pirogov, D. V. Denisov, and A. Yu. Egorov. Photoluminescence of heterostructures with $\mathrm{GaP}_{1}$

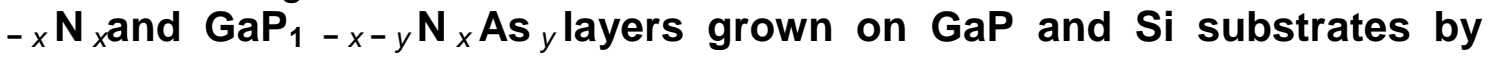
molecular-beam epitaxy. Semiconductors 49, 479 (2015)

13 S. Shokhovets, O. Supplie, C. Koppka, S. Krischok, T. Hannappel. Optical constants and origin of the absorption edge of GaPN lattice-matched to Si. Physical Review B 98:7, 075205. (2018).

14 K. Zelazna, M. Gladysiewicz, M. P. Polak, S. Almosni, A. Létoublon, C. Cornet, O. Durand, W. Walukiewicz, R. Kudrawiec. Nitrogen-related intermediate band in P-rich $\mathrm{GaN}_{\mathbf{x}} \mathbf{P}_{\mathbf{y}} \mathbf{A s}_{\mathbf{1 - x}-\mathbf{y}}$ alloys. Sci Rep 7, Article number: 15703 (2017).

15 Antonio L. Luque, Antonio Zabalza Martí. The intermediate band solar cell: progress toward the realization of an attractive concept. Adv. Mater. 2010, 22, 160-174.

16 J. Wu, W. Shan, and W. Walukiewicz, Semicond. Band anticrossing in highly mismatched III-V semiconductor alloys. Sci. Technol. 17, 860 (2002).

17 Buyanova IA, Chen WM, Tu CW. Defects in dilute nitrides. J Phys Condens Matter 16:S3027-S3035(2004).

18 Chen WM, Buyanova IA, Tu CW, Yonezu H. Point defects in dilute nitride III-N-As and III-N-P. Phys B Condens Matter 376-377:545-551(2006).

19 Geisz JF, Reedy RC, Keyes BM, Metzger WK Unintentional carbon and hydrogen incorporation in GaNP grown by metal-organic chemical vapor deposition. J Cryst Growth 259:223-231(2003).

20 Jussila, H, Yu, K.M, Kujala, J, Tuomisto, F, Subramaniyam, Nagarajan, Lemettinen, J, Huhtio, T, Tuomi, T.O, Lipsanen, H. Substitutionality of nitrogen atoms and formation of nitrogen complexes and point defects in GaPN alloys. Sopanen, M . J. Phys. D:Appl. Phys. 47075106 (2014).

21. Y. Jin, R.M. Jock, H. Cheng, Y. He, A.M. Mintarov, Y. Wang, C. Kurdak, J.L. Merz, and R.S. Goldman. Influence of $\mathbf{N}$ interstitials on the electronic properties of GaAsN alloys. Appl. Phys. Lett. 95, 062109 (2009).

22 Y.-J. Kuang, S.-W. Chen, H. Li, S.K. Sinha, and C.W. Tu, J. Growth of $\mathrm{GaN}_{\mathrm{x}} \mathrm{As} \mathrm{s}_{\mathrm{y}} \mathrm{P}_{1-\mathrm{x}-\mathrm{y}}$ alloys on $\mathrm{GaP}(100)$ by gas-source molecular beam epitaxy . Journal of Vacuum Science \& Technology B 30, 02B121 (2012) 
23. T. Quinci a,b , J. Kuyyalil a , T. Nguyen Thanh a , Y. Ping Wang a, S. Almosni a , A. Létoublon a , T. Rohel a , K. Tavernier a , N. Chevalier a , O. Dehaese a , N. Boudet c , J.F. Bérar c , S. Loualiche a , J. Even a, N. Bertru a, A. Le Corre a , O. Durand a , C. Cornet a. Defects limitation in epitaxial GaP on bistepped Si surface using UHVCVD-MBE growth cluster. J. Cryst. Growth 380, 157 (2013).

24. C. Cornet, T. Nguyen Thanh, T. Quinci, S. Almosni, T. Rohel, J. Kuyyalil, A. Rambaud, A. Létoublon, N. Bertru, O. Durand, and A. Le Corre. Preferential incorporation of substitutional nitrogen near the atomic step edges in diluted nitride alloys. Applied Physics Letters 101, 251906 (2012).

25 W. Shan, W. Walukiewicz, K. M. Yu, and J. W. Ager III. Nature of the fundamental band gap in GaNxP1-xGaNxP1-x alloys. Appl. Phys. Lett. 76, 3251 (2000).

26 W. Shan, W. Walukiewicz, J. W. Ager, E. E. Haller, J. F. Geisz, D. J. Friedman, J. M. Olson, and S. R. Kurtz. Band Anticrossing in GalnNAs Alloys. Phys. Rev. Lett. 82, 1221 (1999).

27 W. Walukiewicz, W. Shan, J. W. Ager III, D. R. Chamberlin, E. E. Haller, J. F. Geisz, D. J. Friedman, J. M. Olson, and S. R. Kurtz. Nitrogen-induced modification of the electronic strucutreof group III-V-N alloys. Proc.Electrochem. Soc. 99-11, 190 (1999).

28 W. Shan, K. M. Yu, W. Walukiewicz, J. W. Ager III, E. E. Haller, and M. C. Ridgeway. Reduction of band-gap energy in GaNAs and AIGaNAs synthesized by implantation. Appl. Phys. Lett. 75, 1410 (1999).

29 C. Robert, M. Perrin, C. Cornet, J. Even, and J. M. Jancu. Atomistic calculations of $\mathrm{Ga}(\mathrm{NAsP}) / \mathrm{GaP}(\mathrm{N})$ quantum wells on silicon substrate: Band structure and optical gain. Appl. Phys. Lett. 100, 111901 (2012).

30 A. Luque and A. Marti. The intermediate band solar cell: Progress toward the realization of an attractive concept. Adv. Mater. 22, 160-174 (2010)

31 T. Sogabe, Y. Shoji, M. Ohba, K. Yoshida, R. Tamaki, H-F. Hong, C-H. Wu, C-T. Kuo, S. Tomic, and Y. Okada, Intermediate-band dynamics of quantum dots solar cell in concentrator photovoltaic modules. Sci. Rep. 4, 4792 (2014).

32 N. Kamata, M. Suetsugu, D. Haque, S. Yagi, H. Yaguchi, F. Karlsson, and P. O. Holtz. Spectral change of intermediate band luminescence in GaP:N due to below-gap excitation: Discrimination from thermal activation. Phys. Status Solidi B 254, No. 2, 1600566 (2017).

33 C. Karcher, K. Jandieri, B. Kunert, R. Fritz, M. Zimprich, K. Volz, W. Stolz, F. Gebhard, S. D. Baranovskii, and W. Heimbrodt. 
Peculiarities of the photoluminescence of metastable $\mathrm{Ga}(\mathrm{N}, \mathrm{As}, \mathrm{P}) / \mathrm{GaP}$ quantum well structures. Phys. Rev. B 82, 245309 (2010).

34. Q. Li, S.J. Xu, M.H. Xie, and S.Y. Tong. Origin of the 'S-shaped' temperature dependence of luminescent peaks from semiconductors . J. Phys. Condens. Matter 17, 4853 (2005).

35 M. Latkowska, R. Kudrawiec, F. Janiak, M. Motyka, J. Misiewicz, Q. Zhuang, A. Krier, and W. Walukiewicz. Temperature dependence of photoluminescence from InNAsSb layers: The role of localized and free carrier emission in determination of temperature dependence of energy gap. Appl. Phys. Lett. 102, 122109 (2013).

36 Taiping Lu, Ziguang Ma, Chunhua Du, Yutao Fang, Haiyan Wu, Yang Jiang, Lu Wang, Longgui Dai, Haiqiang Jia, Wuming Liu, Hong Che . Temperature-dependent photoluminescence in light-emitting diodes. Sci. Rep. 4, 6131 (2014).

37 Zilan Wang, Lai Wang, Yuchen Xing, Di Yang, Jiadong Yu, Zhibiao Hao, Changzheng Sun, Bing Xiong, Yanjun Han, Jian Wang, Hongtao Li, and $\mathrm{Yi}$ Luo. Consistency on Two Kinds of Localized Centers Examined from Temperature-Dependent and Time-Resolved Photoluminescence in InGaN/GaN Multiple Quantum Wells. ACS Photonics (2017) ,4, 8, 20782084.

38 V K Dixit, S Porwal, S D Singh, $T$ K Sharma, Sandip. A Versatile Phenomenological Model For The S-Shaped Temperature Dependence Of Photoluminescence Energy For An Accurate Determination Of The Exciton Localization Energy In Bulk And Quantum Well Structures. J. Phys. D: Appl. Phys. 47, 065103 (2014).

39 O. Rubel, M. Galluppi, S. D. Baranovskii, K. Volz, L. Geelhaar, H. Riechert, P. Thomas, and W. Stolz. Quantitative description of disorder parameters in (Galn)(NAs) quantum wells from the temperature-dependent photoluminescence spectroscopy. Journal of Applied Physics 98, 063518 (2005).

40 S. Francoeur, G. Sivaraman, Y. Qiu, S. Nikishin, And H. Temkin, Luminescence of As-Grown And Thermally Annealed GaAsN/GaAs. Appl. Phys. Lett. 72, 1857 (1998).

41 P H Borcherds, G F Alfrey, A D B Woods and D H Saunderson. Phonon dispersion curves in indium phosphide. J. Phys. C: Solid State Phys. 82022 (1975).

42 Grieb T, Müller K, Fritz R, Grillo V, Schowalter M, Volz K, Rosenauer A. Quantitative chemical evaluation of dilute GaNAs using ADF STEM: avoiding surface strain induced artifacts. Ultramicroscopy 129, 1-9 (2013).

43 Grieb T, Müller K, Fritz R, Schowalter M, Neugebohrn N, Knaub N, Volz $\mathrm{K}$, Rosenauer A. Determination of Nitrogen Concentration in Dilute GaNAs 
by STEM HAADF Z-Contrast Imaging and improved STEM-HAADF strain state analysis. Ultramicroscopy 117, 15-23 (2012).

44. V. Grillo, E. Carlino, and F. Glas. Influence of the static atomic displacement on atomic resolution Z-contrast imaging. Phys. Rev. B 77, 054103 (2008).

45 D. G. Thomas and J. J. Hopfield. Isoelectronic Traps Due to Nitrogen in Gallium Phosphide. Phys. Rev. 150, 680 (1966)

46 M. Izadifard, J. P. Bergman, I. Vorona, W. M. Chen, and I. A. Buyanova. Evaluation of optical quality and defect properties of GaNxP1-x alloys lattice matched to Si. Appl. Phys. Lett. 85, 6347 (2004).

47 A Utsumi , H. Yonezu, Y. Furukawa, k. Momose, K. Kuroki. Increase in luminescence efficiency of GaPN layers by thermal annealing. Phys. Stat. Sol. (C) 0, no. 7, 2741-2744 (2003).

48 Hopil P. Bae, Seth R. Bank, Homan B. Yuen, Tomas Sarmiento, Evan R. Pickett, Mark A. Wistey $D$, James $S$ Harris. Temperature dependencies of Annealing behaviors of GalnNAsSb/GaNAs quantum wells for long Wavelength dilute-nitride lasers. Applied physics letters 90, 231119 (2007).

49 S.L. Tan, C.J. Hunter, S. Zhang, L.J.J. Tan, Y.L. Goh, J.S. N g, I.P. Marko, S.J. Sweeney, A.R. Adams, j. Allam, J.P.R. David. Improved. Optoelectronic properties of rapid thermally annealed dilute nitride GalnNAs photodetectors. Journal of electronic materials, vol. 41, No. 12, (2012).

50 K. Volz, J. Koch, B. Kunert, I. Nemeth, w. Stolz. Influence of annealing on The optical and structural properties of dilute n-containing III/V. Semiconductor heterostructures. Journal of crystal growth volume 298, (2007), pages 126-130.

51 Balanta, M.A.G, Orsi Gordo, V, Carvalho, A.R.H, Puustinen, J, Alghamdi, H.M., Henini, M., Galeti, H.V.A., Guina, M, Galvão Gobato, Y. Polarization resolved photoluminescence in GaAs1-xBix/GaAs quantum wells. Journal of Luminescence, v. 182, p. 49-52 (2017).

52 Balanta, M A G, Kopaczek, J, Orsi Gordo, V, Santos, B H B, Rodrigues, A D, Galeti, H V A, Richards, R D, Bastiman, F, David, J P R, Kudrawiec, R, Galvão Gobato, $Y$. Optical and spin properties of localized and free excitons in GaBiAs /GaAs multiple quantum wells. Journal of Physics. D, Applied Physics (Print), v. 49, p. 355104 (2016).

53 Ya. V. Terent'ev, S. N. Danilov, J. Loher, D. Schuh, D. Bougeard, D. Weiss, M. V. Durnev, S. A. Tarasenko, M. S. Mukhin, S. V. Ivanov, and S. D. Ganichev. Magneto-photoluminescence of InAs/InGaAs/InAlAs Quantum Well Structures. Applied Physics Letters 104, 101111 (2014).

54 F. Meier and B. P. Zakharchenya. Optical Orientation (Elsevier Science, Amsterdam, 1984). 
55 L. Lombez, P. F. Braun, H. Carrère, B. Urbaszek, P. Renucci, T. Amand, X. Marie, J. C. Harmand, And V. K. Kalevich. Spin Dynamics in Dilute Nitride Semiconductors At Room Temperature. Applied Physics Letters 87, 252115 (2005).

56 F. Zhao, A Balocchi, G Truong, T Amand, X Marie, X J Wang, I A Buyanova, W M Chen, J C Harmand. Electron Spin Control in Dilute Nitride Semiconductors. J. Phys: Condens. Matter 21 (2009) 174211 (5pp).

57 Skierbiszewski, P. Pfeffer, and J. Łusakowski . Effective g- Factor In The Diluted Nitrides $\mathbf{G a}_{1-y} \operatorname{In}_{\mathbf{y}} \mathbf{N}_{\mathbf{x}} \mathbf{A s}_{1-\mathrm{x}}$. Phys. Rev. B 71, 205203 (2005). 


\section{Figure Captions}

Figure 1: (a) Typical PL specta at $300 \mathrm{~K}$ for GaPN samples with various $\mathrm{N}$ compositions (b) Energy of the maximum room temperature PL intensity as a function of $\mathrm{N}$ content at room temperature. The red solid line is the calculated bandgap using tight-binding models from ref. [29]

Figure 2: Typical $\mathrm{PL}$ spectra at low temperature $(\mathrm{T}=10 \mathrm{~K})$ for different laser powers. (a) $\mathrm{N}=2 \%$ and (b) $\mathrm{N}=2.7 \%$

Figure 3. PL peak energy versus $T$ (solid circles) and Vashni fitting (solid lines) at $30 \mathrm{~mW}$. (a) as-grown sample, (b) after annealing.

Figure 4: PL spectra at $300 \mathrm{~K}$ before and after thermal annealing for a laser wavelength of $488 \mathrm{~nm}$ and power of $100 \mathrm{~mW}$.

Figure 5: (a) Raman spectra at $300 \mathrm{~K}$ for samples with different $\mathrm{N} \%$ incorporation using a laser of $488 \mathrm{~nm}(2.54 \mathrm{eV})$. The inset shows the details of the LO-mode peak. (b) Raman spectra of the as-grown and annealed samples with $\mathrm{N}=2.1 \%$,.

Figure 6: STEM-HAADF images for a $\mathrm{GaP}_{0.98} \mathrm{~N}_{0.02}$ sample (a) as-grown and (b) annealed. A comparison of HAADF intensity profiles normalized to the one of $\mathrm{GaP}$ is given in (c).

Figure 7. (a) Typical PL and PLE for $\mathrm{N}=1.4 \%$ at $10 \mathrm{~K}$ for as-grown and annealed samples. (b) Stokes shift (c) edge and threshold energy (left) and band width dependence with the nitrogen concentration for annealed samples (right).

Figure 8. Typical Streak camera images for $\mathrm{N}=1 \%$ at $10 \mathrm{~K}$ for (a) as-grown and (b)annealed samples. The temperature dependence of the measured PL decay times of the $1 \% \mathrm{~N}$ containing GaP sample as grown (c) and after annealing (d)

Figure 9: Decay times measured in the $\mathrm{GaP}_{0.98} \mathrm{~N}_{0.02}$ sample as a function of temperature for (a) as-grown sample and (b) annealed sample. Associated Boltzmann plots are drawn in (c), with corresponding extracted activation energies. PL linewidths as function of temperature are given in (d).

Figure 10: Magnetic dependence of the PL spectra for the $\mathrm{N}=2.1 \%$ sample. The inset shows the normalized spectra for $0 \mathrm{~T}$ and $15 \mathrm{~T}$. Measurements taken at $2 \mathrm{~K}$ with a power excitation of $30 \mathrm{~mW}$.

Figure 11. Polarization resolved spectra obtained at $2 \mathrm{~K}, 15 \mathrm{~T}$ at and power excitation of $25 \mathrm{~mW}$ for both (a) as-grown and (b) annealed GaPN samples with $\mathrm{N}=2.1 \%$. The inset shows the normalized spectra for $\sigma+$ and $\sigma-$ emission.

Figure 12. Magnetic field dependence of polarization degree at $2 \mathrm{~K}$ before and after thermal annealing for GaPN layers for different N\% contents 
Figure 1
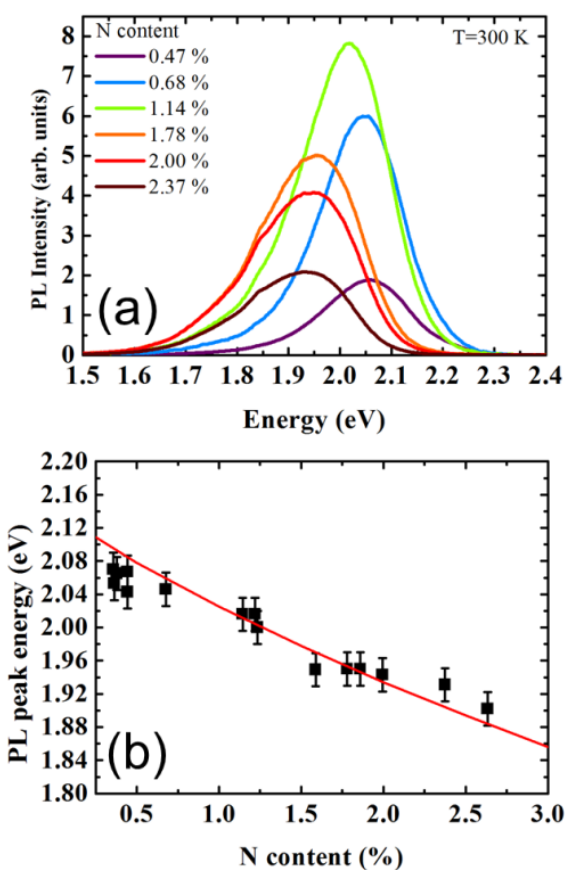

Figure 2.
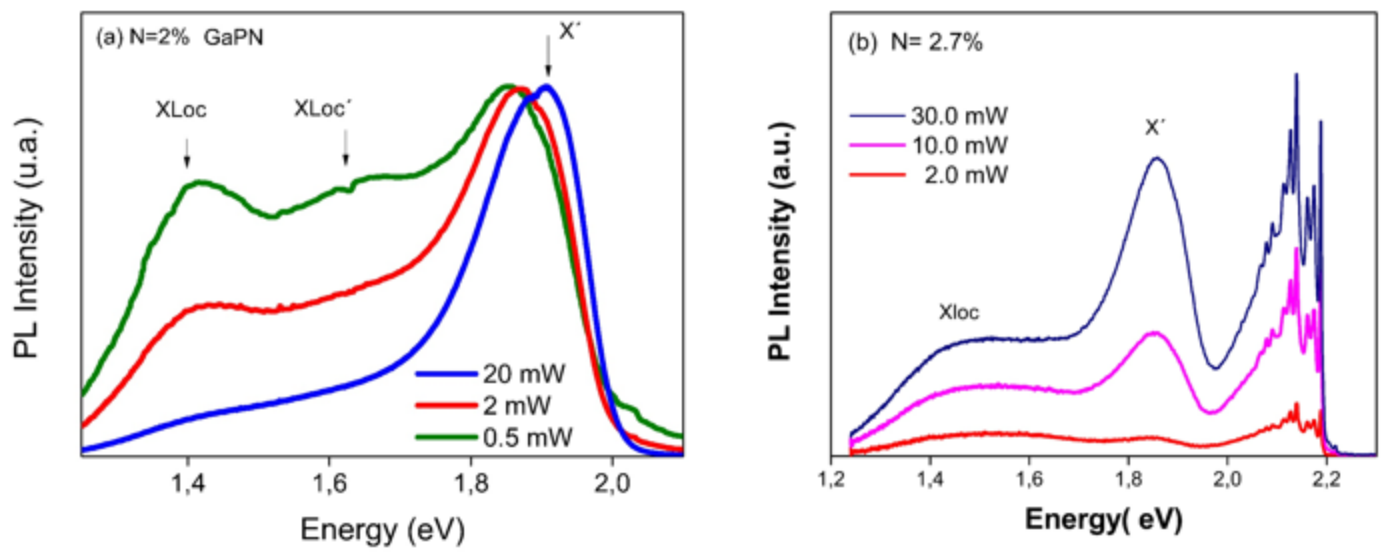
Figure 3
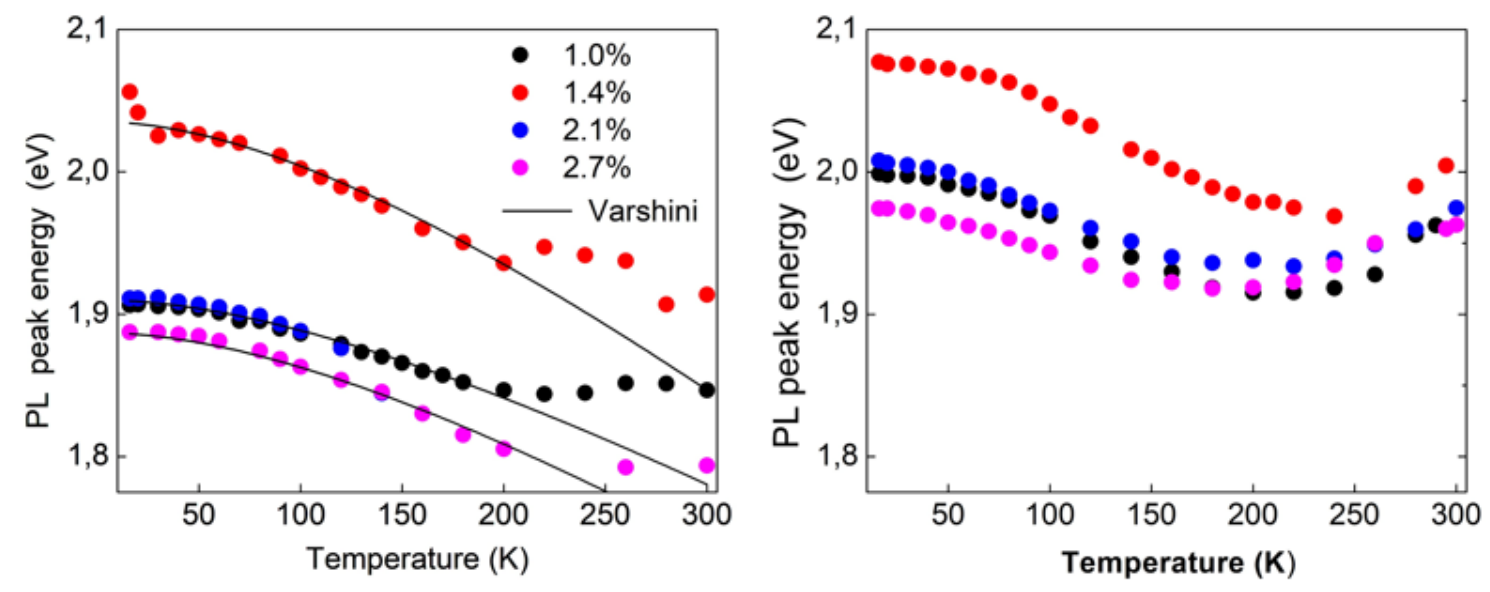

Figure 4

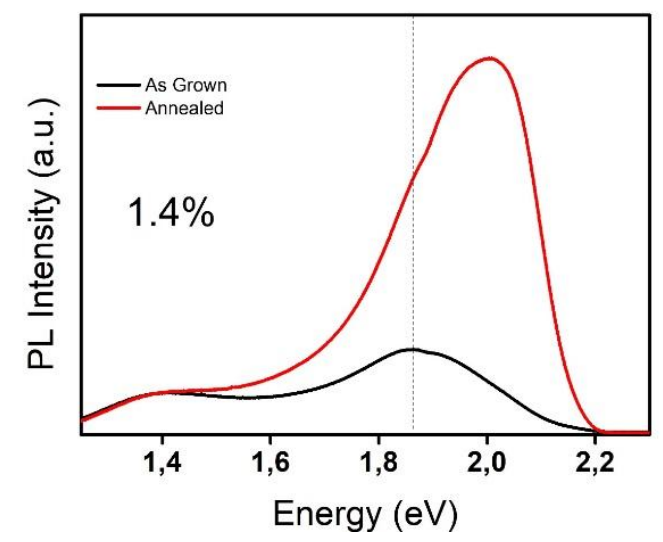

Figure 5
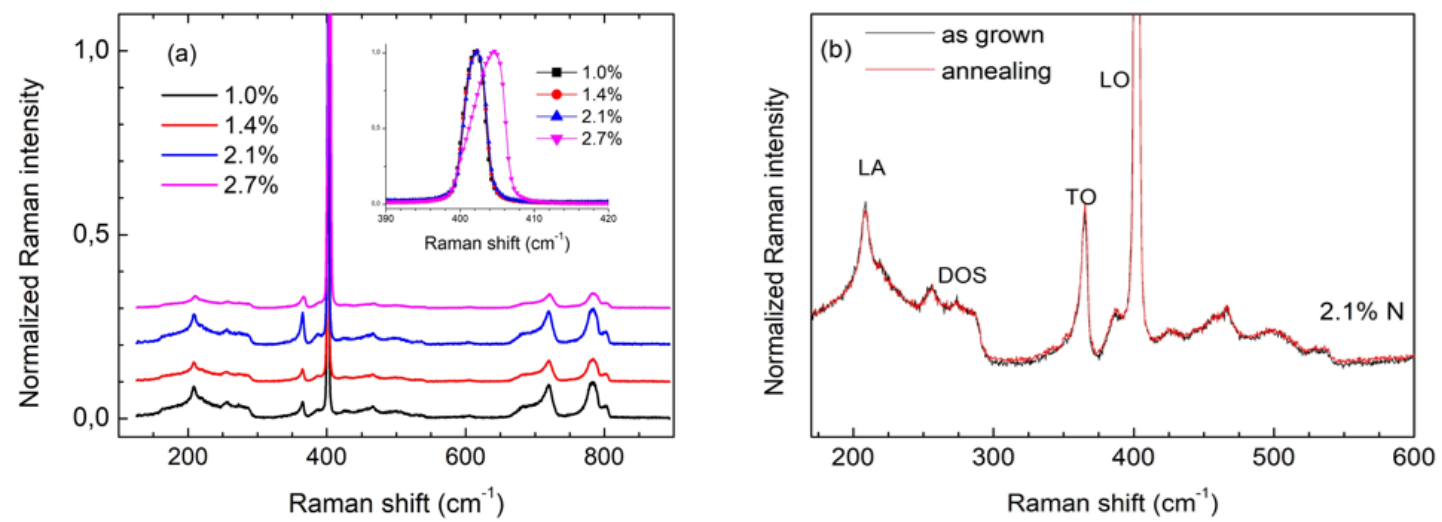
Figure 6
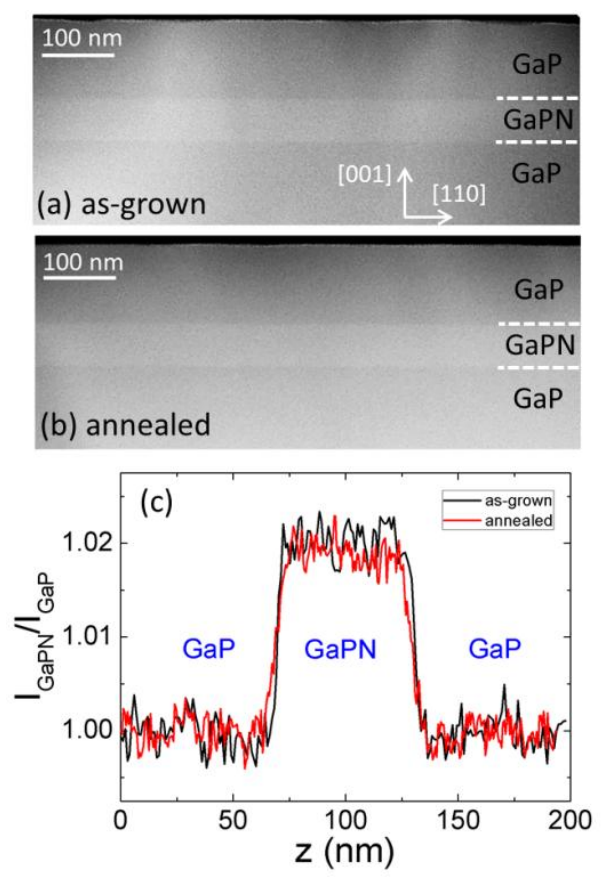

Figure 7
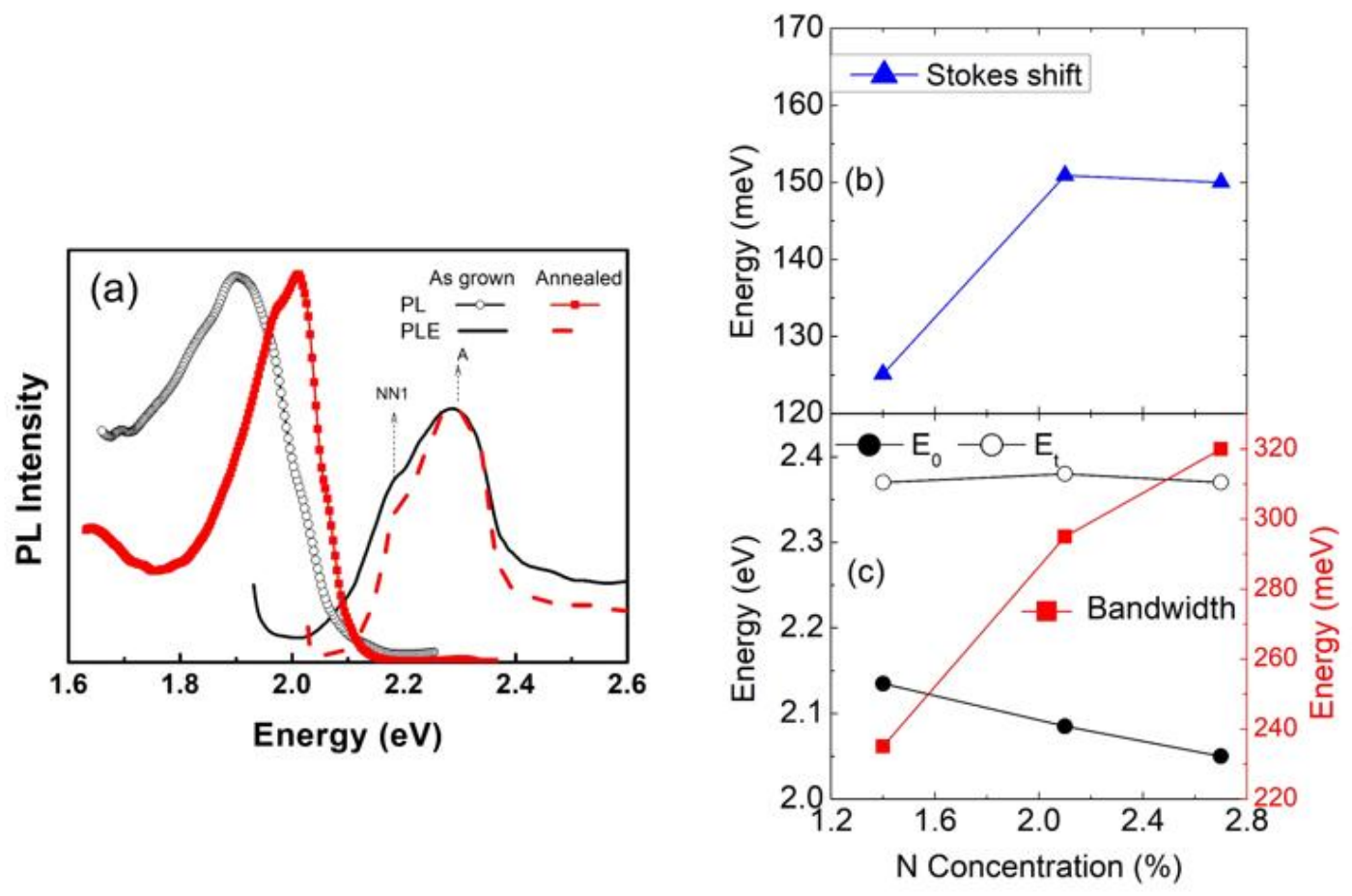
Figure 8
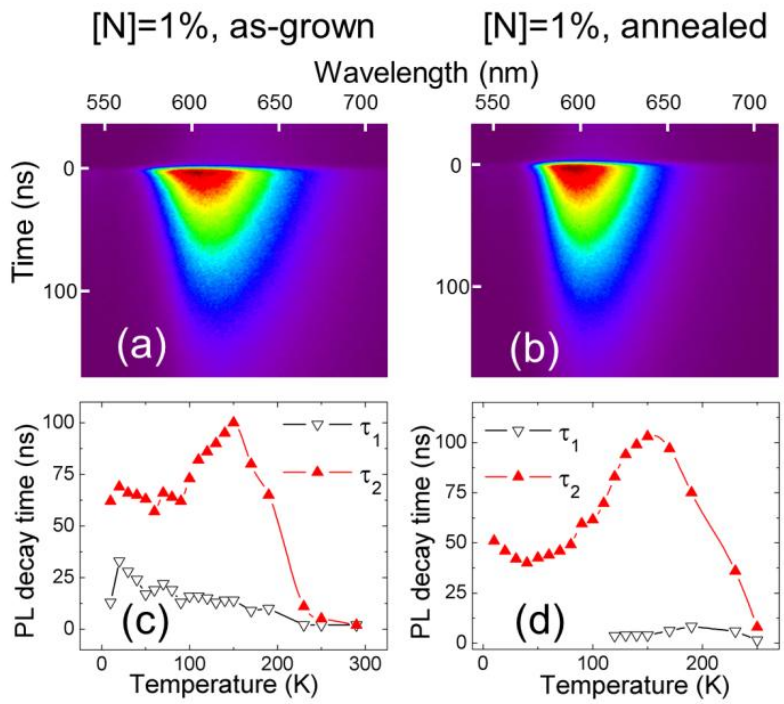

Figure 9
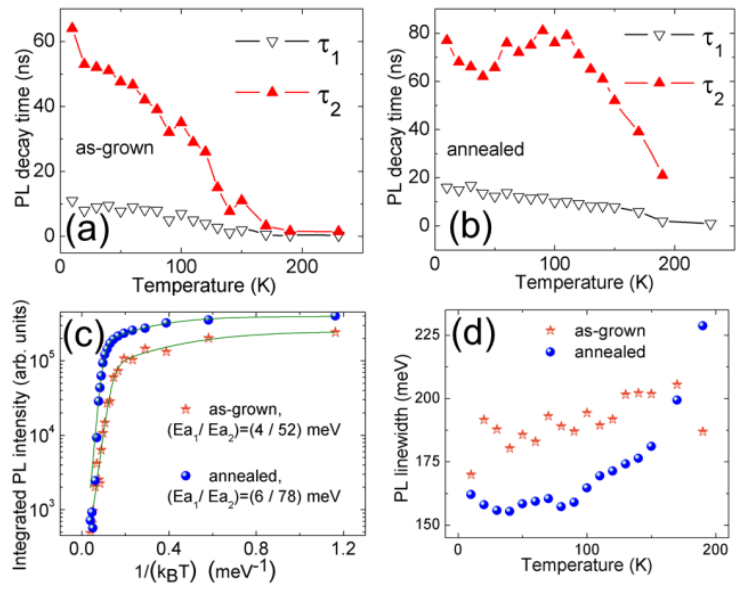
Figure 10

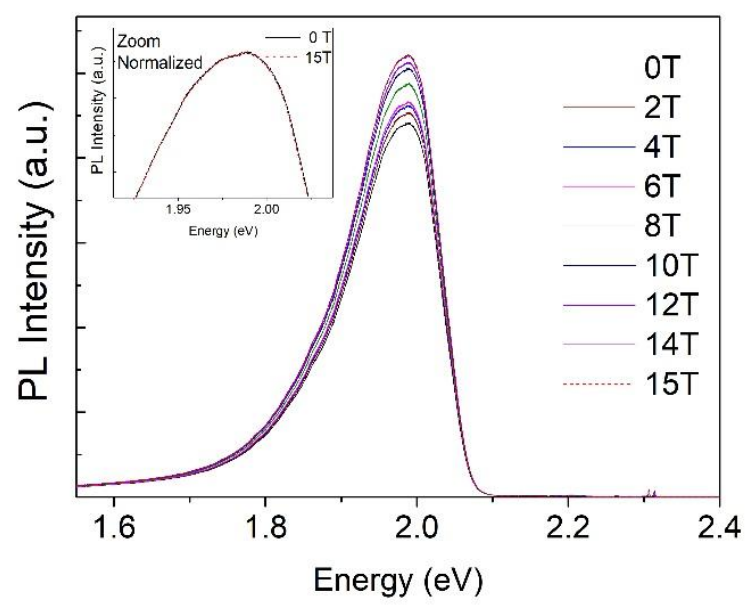

Figure 11 


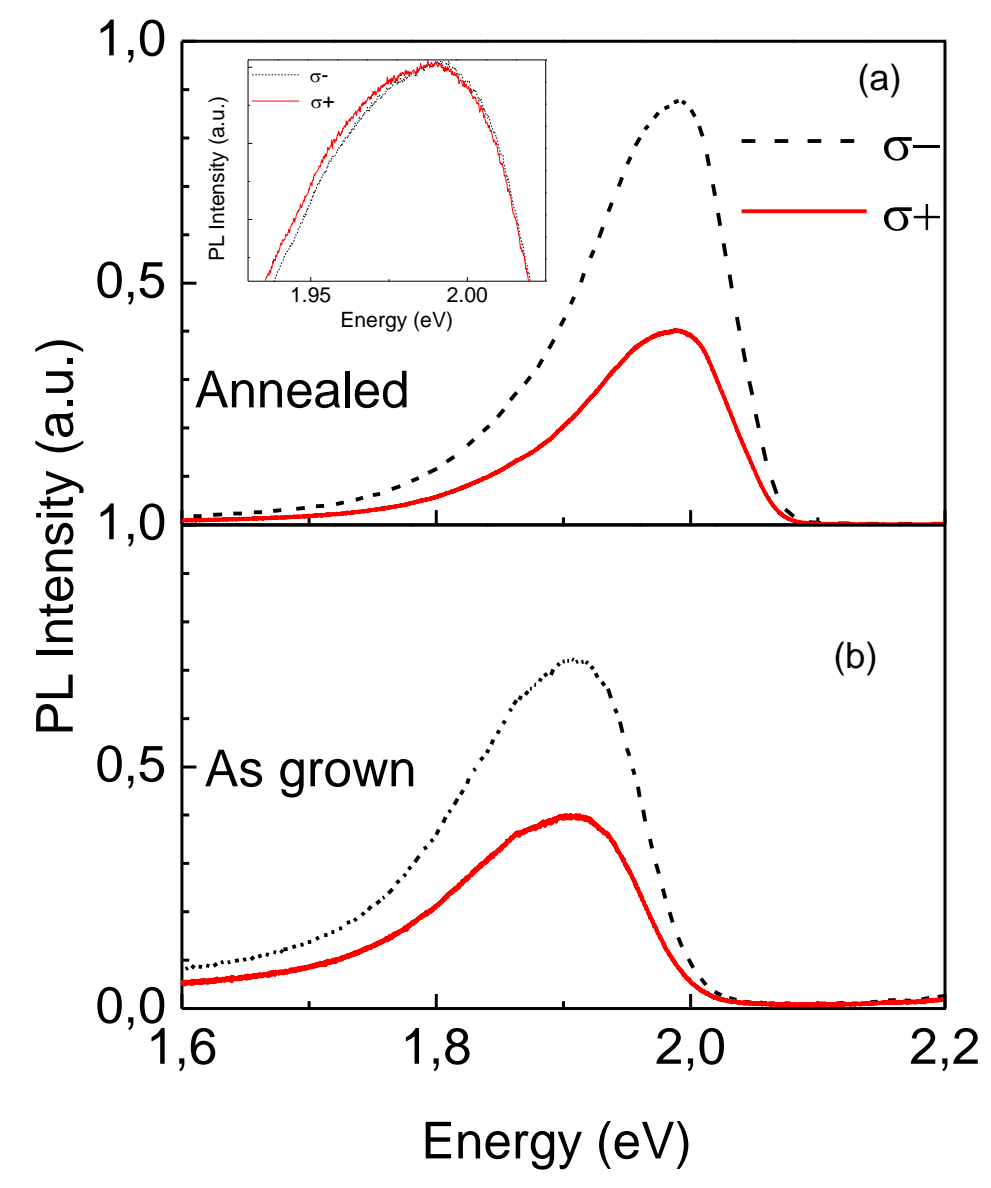

Figure 12 


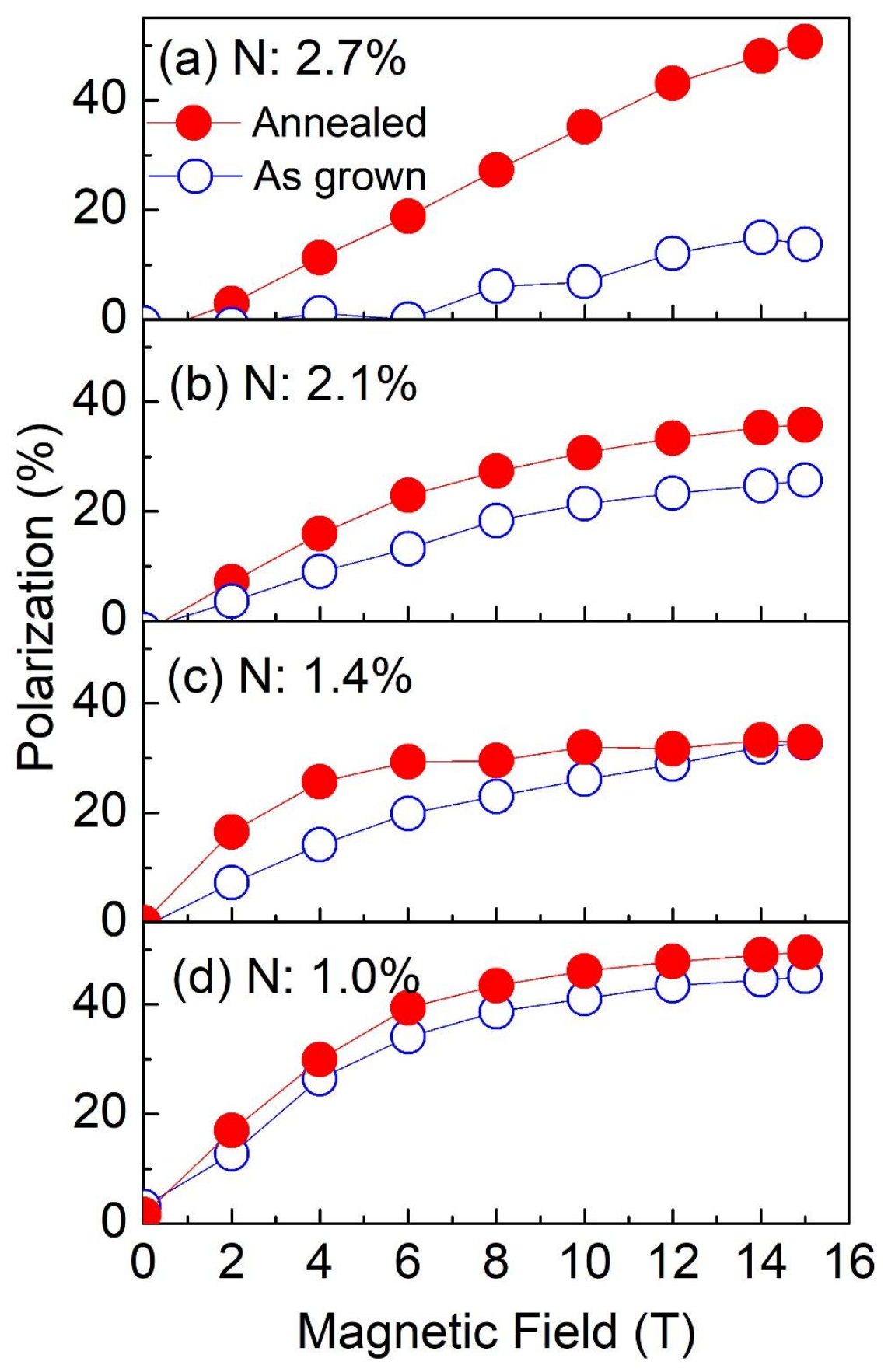

